\title{
Critical case for singularly perturbed linear boundary-value problems of ordinary differential equations
}

\author{
Ljudmil Karandzhulov
}




\title{
CRITICAL CASE FOR SINGULARLY PERTURBED LINEAR BOUNDARY-VALUE PROBLEMS OF ORDINARY DIFFERENTIAL EQUATIONS
}

\author{
LYUDMIL KARANDZHULOV
}

[Received: May 12, 2003 and, in revised form, March 21, 2006]

\begin{abstract}
The conditions under which a unique asymptotic representation of the solution of boundary-value problems exists for singularly perturbed systems of ordinary differential equations are shown in the work. The solution is obtained with the help of boundary functions and pseudo-inverse matrices.
\end{abstract}

Mathematics Subject Classification: 34B15

Keywords: boundary-value problems, singularly perturbation, asymptotic solution, boundary functions

\section{Statement of the Problem}

We consider the singularly perturbed differential system

$$
\begin{gathered}
\varepsilon \dot{x}=A x+\varepsilon A_{1}(t) x+\varphi(t), \quad t \in[a, b], \quad 0<\varepsilon \ll 1, \\
l x(\cdot)=h, \quad h \in \mathbb{R}^{m},
\end{gathered}
$$

where the coefficients of system (1.1) and equation (1.2) satisfy the conditions:

(H1) $A$ is a constant $(n \times n)$ matrix. If $\lambda_{i}$ are eigenvalues of $A$, then $\lambda_{i}=0, i=\overline{1, k}$, $k<n, \operatorname{Re} \lambda_{i}<0, i=\overline{k+1, n}$, as $p, p<k$, linear independent eigenvectors of matrix $A$ correspond to the zero eigenvalue;

(H2) $A_{1}(t)$ is an $(n \times n)$ matrix, $A_{1}(t) \in C^{\infty}[a, b], \varphi(t)$ is an $n$-dimensional vectorfunction $\varphi(t) \in C^{\infty}[a, b]$

(H3) $l: C[a, b] \rightarrow \mathbb{R}^{m}$ is an $m$-dimensional linear bounded vector-functional, $l=\operatorname{col}\left(l^{1}, \ldots, l^{m}\right)$

(H4) The degenerate $(\varepsilon=0)$ system $(1.1), A x_{0}+\varphi(t)=0$, is solvable with respect to $x_{0}$.

We look for an $n$-dimensional vector-function $x(t, \varepsilon): x(\cdot, \varepsilon) \in C^{1}[a, b], x(t, \cdot) \in$ $C\left(0, \varepsilon_{0}\right]$, satisfying (1.1), (1.2) and following relation $\lim _{\varepsilon \rightarrow 0} x(t, \varepsilon)=x_{0}(t), t \in(a, b]$. 
We shall consider the case $m \neq n$ and $p<k$. We use an asymptotic method of the boundary functions and construct an asymptotic series for the boundary-value problem (1.1), (1.2) with $\operatorname{det} A=0$ (the critical case [10]).

In the case $m=n$ and $p=k$ an asymptotic solution of the Cauchy problem and two point boundary-value problem for linear and quasilinear systems is studied in [10] on the basis of the method of boundary functions. In the non-critical case $m \neq n$ and $\operatorname{det} A \neq 0$ the system is studied in [5]. When $m \neq n$ and $p=k$, the problem (1.1), (1.2) is considered in [8].

The construction of an asymptotic solution of (1.1), (1.2) in this work $m \neq n, p<k$ is represented on the basis of generalized inverse matrices and projectors $[1,4,7]$ and central canonical form $[2,3]$.

We denote by $n_{1}, n_{2}, \ldots, n_{p}\left(\sum_{i=1}^{p} n_{i}=k\right)$ the lengths of the Jordan cells. We will consider the case where $n_{1}>\cdots>n_{s}, n_{s+1}=n_{s+2}=\cdots=n_{p-1}=n_{p}=1$, i. e., the matrix $A$ has a block diagonal representation

$$
A=\operatorname{diag}\left(\bar{A}, J_{1}, J_{2}, \ldots, J_{s}, \Theta_{p-s}\right),
$$

where $\bar{A}$ is a $((n-k) \times(n-k))$ matrix and has eigenvalues with negative real parts, $J_{i}, i=\overline{1, s}$, are $\left(n_{i} \times n_{i}\right)$ Jordan cells, and $\Theta_{p-s}$ is the $((p-s) \times(p-s))$ zero matrix.

By $A^{\dagger}$, we denote the unique Moore-Penrose pseudo-inverse $(n \times n)$ matrix of the matrix $A[4,7]$. Denote by $P_{A}$ and $P_{A^{*}}$ orthoprojectors $P_{A}: \mathbb{R}^{n} \rightarrow \operatorname{ker} A, P_{A^{*}}: \mathbb{R}^{n} \rightarrow$ $\operatorname{ker} A^{*}, A^{*}=A^{T}$. According to (H1) we find $\operatorname{rank} A=n-p$ and $\operatorname{rank} P_{A}=\operatorname{rank} P_{A^{*}}=$ $n-(n-p)=p$. Let $P_{A_{p}}$ be a $(n \times p)$ matrix with $p$ linear independent columns from the matrix $P_{A}$, and let $P_{A_{p}^{*}}$ be a $(p \times n)$ matrix with $k p$ linear independent rows of the matrix $P_{A^{*}}$.

Let $C=P_{A_{p}^{*}} P_{A_{p}}$ be an $(m \times n)$-constant matrix.

Lemma 1. $\operatorname{rank} C=p-s$.

Proof. The proof is based on the equalities $J_{i} J_{i}^{\dagger}=\operatorname{diag}(1,1, \ldots, 1,0)$ and $J_{i}^{\dagger} J_{i}=$ $\operatorname{diag}(0,1, \ldots, 1,1)$. Keeping in mind the representation

$$
A^{\dagger}=\operatorname{diag}\left(\bar{A}^{-1}, J_{1}^{\dagger}, J_{2}^{\dagger}, \ldots, J_{s}^{\dagger}, \Theta_{p-s}\right)
$$

and the equalities $P_{A}=E_{n}-A^{\dagger} A, P_{A^{*}}=E_{n}-A A^{\dagger}$, we get that $C=P_{A_{p}^{*}} P_{A_{p}}=$ $\operatorname{diag}\left(0, E_{p-s}\right)$, i. e., $\operatorname{rank} C=p-s$.

We consider the degenerate differential system

$$
C \frac{d}{d t} z(t)=B(t) z(t)+l(t), \quad t \in[a, b]
$$

where $C$ is the matrix from Lemma 1.1, $B(t)=P_{A_{p}^{*}} A_{1}(t) P_{A_{p}}$ is $(p \times p)$ matrix, and $l$ is a $p$-dimensional vector-function, $l(t) \in C^{\infty}[a, b]$. 
Let the matrix $B(t)$ have the block representation

$$
\left(\begin{array}{ll}
B_{11}(t) & B_{12}(t) \\
B_{21}(t) & B_{22}(t)
\end{array}\right)
$$

where the matrices $B_{11}, B_{12}, B_{21}$, and $B_{22}$ have dimensions $((p-s) \times(p-s)),((p-$ $s) \times s),(s \times(p-s))$, and $s \times s$, respectively.

Lemma 2. System (1.4) takes the central canonical form if and only if $\operatorname{det} B_{11} \neq 0$ $\forall t \in[a, b]$.

Proof. The proof of Lemma 2 is based on Lemma 1 and the work [3].

In accordance with Lemma 1 under $p \neq s$ and Lemma $2(p \times p)$, matrices $P(t)$ and $Q(t)$ exist such that substituting $z(t)=Q(t) y(t)$ and multiplying by $P(t)$ on the left, the system (1.4) takes central canonical form

$$
\left(\begin{array}{cc}
E_{p-s} & 0 \\
0 & \Theta_{s}
\end{array}\right) \frac{d y(t)}{d t}=\left(\begin{array}{cc}
L(t) & 0 \\
0 & E_{s}
\end{array}\right) y(t)+\left(\begin{array}{c}
\mu(t) \\
v(t)
\end{array}\right)
$$

where $\Theta_{s}$ is the $(s \times s)$ zero matrix, $L(t)$ is a $((p-s) \times(p-s))$ matrix, $E_{p-s}$ and $E_{s}$ are $((p-s) \times(p-s))$ and $(s \times s)$ unit matrices, respectively, and $\mu(t)$ and $v(t)$ are $(p-s)$ and $s$-dimensional vector-functions such that

$$
P(t) g(t)=\left(\begin{array}{c}
\mu(t) \\
v(t)
\end{array}\right)
$$

Let the $(p-s)$-dimensional vector-function $u(t)$ and $s$-dimensional vector-function $v(t)$ are such that $y(t)=\left(\begin{array}{l}u(t) \\ v(t)\end{array}\right)$. Then the system (1.5) takes the form

$$
\begin{aligned}
\dot{u}_{i}(t) & =L(t) u_{i}(t)+\mu_{i}(t), \\
0 & =v_{i}(t)+v_{i}(t) .
\end{aligned}
$$

We denote by $\Phi(t)$ a normal fundamental matrix of the solutions of the system $\dot{x}=$ $L(t) x$. Then system (1.7) has a generalized solution

$$
\begin{aligned}
& u(t)=\Phi(t) \Phi^{-1}(t) \eta+\bar{u}(t), \quad \eta \in \mathbb{R}^{\mathbf{p}-\mathbf{s}}, \\
& v(t)=-v(t),
\end{aligned}
$$

where $\bar{u}(t)=\Phi(t) \int_{a}^{t} \Phi^{-1}(s) \mu(s) d s$.

Let the matrix $Q(t)$ be reduced to the block form $Q(t)=\left[Q_{1}(t), Q_{2}(t)\right]$, where $Q_{1}(t)$ is a $(p \times(p-s))$ matrix and $Q_{2}(t)$ is a $(p \times s)$ matrix. Keeping in mind the substitution $z(t)=Q(t) y(t)$, where $y(t)=[u(t), v(t)]^{T}$, we obtain

$$
z(t)=Q_{1}(t) u(t)+Q_{2}(t) v(t) .
$$

In the last equality we substitute solution (1.8). Thus,

$$
z(t)=\Phi(t, a) \eta+\bar{z}(t), t \in[a, b], \eta \in \mathbb{R}^{p-s},
$$


where

$$
\begin{gathered}
\bar{\Phi}(t, a)=Q_{1}(t) \Phi(t) \Phi^{-1}(a) \quad \text { is a }(p \times(p-s)) \text { matrix and } \\
\bar{z}(t)=Q_{1}(t) \bar{u}(t)-Q_{2}(t) v(t)
\end{gathered}
$$

The following lemma is needed.

Lemma 3. Let the matrix A satisfy condition (H1), and let the vector-function $f(\tau) \in C[0,+\infty)$ and satisfy the inequality $\|f(\tau)\|<c_{1} e^{-\alpha_{1} \tau}$, where $\tau \geq 0, c_{1}>0$, and $\alpha_{1}>0$. Then there exist positive constants $c$ and $\gamma$ such that the system $d x / d \tau=$ $A x+f(\tau)$ has a particular solution of the form

$$
x(\tau)=\int_{0}^{\infty} K(\tau, s) f(s) d s,
$$

satisfying the inequality $\|x(\tau)\| \leq c \exp (-\gamma \tau), \tau \geq 0$, where

$$
K(\tau, s)= \begin{cases}X(\tau) P X^{-1}(s) & \text { for } 0 \leq s \leq \tau<\infty, \\ -X(\tau)(I-P) X^{-1}(s) & \text { for } 0<\tau<s \leq \infty,\end{cases}
$$

and $P$ is the spectral projector of the matrix $A$ to the left semi-plane.

The lemma is proved analogously to a similar lemma in [5].

\section{Formally ASYMPTOTIC EXPANSION}

We shall seek for a formally asymptotic expansion of the solution of problem (1.1), (1.2) in the form of the regular and singular series

$$
x(t, \varepsilon)=\sum_{i=0}^{\infty} \varepsilon^{i}\left(x_{i}(t)+\Pi_{i}(\tau)\right), \quad \tau=\frac{t-a}{\varepsilon},
$$

where $x_{i}(t)$ and $\Pi_{i}(\tau)$ are unknown $n$ vector functions. By $\Pi_{i}(\tau)$ (see [10]) we denote the boundary function in a neighbourhood of the point $t=a$. They will be constructed so that when $0<\varepsilon \leq \varepsilon_{0}$, the inequalities

$$
\left\|\Pi_{i}(\tau)\right\| \leq \gamma_{i} \exp \left(-\alpha_{i} \tau\right)
$$

where $\gamma_{i}$ and $\alpha_{i}$ are positive constants for $i=0,1,2, \ldots$ and $\tau \geq 0$, hold in $[a, b]$.

Formally, by substituting (2.1) in (1.1), (1.2), for $x_{i}(t)$ we obtain the systems

$$
A x_{i}(t)=f_{i}(t), \quad t \in[a, b], \quad i=0,1, \ldots,
$$

where

$$
f_{i}(t)= \begin{cases}-\varphi(t) & \text { for } i=0, \\ L_{1}\left(x_{i-1}(t)\right) & \text { for } i=1,2, \ldots\end{cases}
$$


and $L_{1}$ is the differential operator $L_{1}(x(t))=\frac{d x(t)}{d t}-A_{1}(t) x$. The boundary functions $\Pi_{i}(\tau)$ are solutions of the boundary problems

$$
\begin{gathered}
\frac{d}{d \tau} \Pi_{i}(\tau)=A \Pi_{i}(\tau)+\psi_{i}(\tau), \quad \tau \in\left[0, \tau_{b}\right], \tau_{b}=\frac{b-a}{\varepsilon}, \\
l\left(x_{i}(\cdot)\right)+l\left(\Pi_{i}\left(\frac{(\cdot)-a}{\varepsilon}\right)\right)= \begin{cases}h & \text { for } i=0, \\
0 & \text { for } i=1,2, \ldots,\end{cases}
\end{gathered}
$$

where

$$
\psi_{i}(\tau)= \begin{cases}0 & \text { for } i=0, \\ \sum_{q=i-1}^{0} \frac{1}{q !} \tau^{q} A_{1}^{(q)}(a) \Pi_{i-1-q}(\tau) & \text { for } i=1,2, \ldots,\end{cases}
$$

We denote the normal fundamental matrix of the solutions of the homogeneous system $\frac{d x}{d \tau}=A x, \tau \in\left[0, \tau_{b}\right]$, by $X(\tau)=\exp (\tau A)$. Let $X_{n-k}(\tau)$ be an $(n \times(n-k))$ matrix with $(n-k)$ columns from the matrix $X(\tau)$, consisting of exponentially small functions (see [8]).

2.1. Obtaining the coefficients $\boldsymbol{x}_{\mathbf{0}}(\boldsymbol{t})$ and $\boldsymbol{\Pi}_{\mathbf{0}}(\tau)$. Consider systems (2.3)-(2.6) for $i=0$. Then the degenerate system

$$
A x_{0}(t)+\varphi(t)=0
$$

is solvable with respect to $x_{0}(t)$ (according to (H4)) if and only if $P_{A^{*}} \varphi(t)=0$ for all $t \in[a, b]$, and it has a solution

$$
x_{0}(t)=P_{A_{p}} \alpha_{0}(t)-A^{\dagger} \varphi(t),
$$

where $\alpha_{0}(t)$ is an arbitrary $p$-dimensional vector-function.

The general solution of system (2.4) has the form

$$
\Pi_{0}(\tau)=X_{n-k}(\tau) c_{0}, \quad c_{0} \in \mathbb{R}^{n-k} .
$$

We define the vector-function $\alpha_{0}(t)$ by obtaining of $x_{1}(t)$. Consider the system $A x_{1}(t)=f_{1}(t)$, where $f_{1}(t)=L_{1}\left(x_{0}(t)\right)$. The latter system has a solution

$$
x_{1}(t)=P_{A_{p}} \alpha_{1}(t)+A^{\dagger} L_{1}\left(x_{0}(t)\right)
$$

if and only if $P_{A_{p}^{*}} L_{1}\left(x_{0}(t)\right)=0$ for all $t \in[a, b]$. Keeping in mind the representation $x_{0}(t)$ from (2.8) and $L_{1}$, we obtain the differential system for $\alpha_{0}(t)$,

$$
C \frac{d}{d t} \alpha_{0}(t)=B(t) \alpha_{0}(t)+g_{0}(t), \quad t \in[a, b],
$$

where $g_{0}(t)=-P_{A_{p}^{*}} L_{1}\left(A^{\dagger} \varphi(t)\right)$. System (2.11) coincides with system (1.4) at $l(t) \equiv$ $g_{0}(t), t \in[a, b]$. Then, according to Lemma 2 and the equality (1.9), we obtain

$$
\alpha_{0}(t)=\Phi(t, a) \eta_{0}+\bar{\alpha}_{0}(t), \quad t \in[a, b], \quad \eta_{0} \in \mathbb{R}^{p-s},
$$


where $\bar{\alpha}_{0}(t)=Q_{1}(t) \bar{u}_{0}(t)-Q_{2}(t) v_{0}(t)$,

$$
\begin{gathered}
\bar{u}_{0}(t)=\Phi(t) \int_{a}^{t} \Phi^{-1}(s) \mu(s)_{0} d s, \\
P(t) g_{0}(t)=\left(\begin{array}{c}
\mu_{0}(t) \\
v_{0}(t)
\end{array}\right),
\end{gathered}
$$

and $\Phi(t, a), Q(t)=\left[Q_{1}(t), Q_{2}(t)\right]$, and $P(t)$ are the matrices from Section 1. The vector-functions $u_{0}(t)$ and $v_{0}(t)$ are solutions of the following system (see (1.7)):

$$
\begin{aligned}
\dot{u}_{0}(t) & =L(t) u_{0}(t)+\mu_{0}(t), \\
0 & =v_{0}(t)+v_{0}(t) .
\end{aligned}
$$

We substitute (2.12) into equality (2.8), and for $x_{0}(t)$ we obtain

$$
x_{0}(t)=P_{A_{p}} \Phi(t, a) \eta_{0}+P_{A_{p}} \bar{\alpha}_{0}(t)-A^{\dagger} \varphi(t) .
$$

Finally, for obtaining the functions $x_{0}(t)$ and $\Pi_{0}(t)$ it is sufficient to determine the vectors $\eta_{0} \in \mathbb{R}^{p-s}$ and $c_{0} \in \mathbb{R}^{n-k}$. In this connection, we use the boundary condition (2.5) for $i=0$, where we substitute (2.13) and (2.9). We obtain the vectors $\eta_{0}$ and $c_{0}$ by the system

$$
D_{0}(\varepsilon) c_{0}+S_{0} \eta_{o}=h_{0}
$$

where $D(\varepsilon)=l X_{n-k}(\cdot)$ is an $(m \times(n-k))$ matrix, $S_{0}=l A_{p} \Phi(\cdot, a)$ is an $(n \times(p-s))$ matrix, $h_{0}=h-l\left(A_{p} \bar{\alpha}_{0}(\cdot)\right)-l\left(A^{\dagger} \varphi(\cdot)\right)$ is an $m$-dimensional vector.

Keeping in mind the expression of the matrix $X_{n-k}(\tau)$ and the form of the functional $l(x)$, we assume that $D_{0}(\varepsilon)=\bar{D}_{0}+O\left(\varepsilon^{s} \exp (-\alpha / \varepsilon)\right)$, where $\alpha>0, s \in N, \bar{D}_{0}$ is a $(m \times(n-k))$-constant matrix, and $O\left(\varepsilon^{s} \exp (-\alpha / \varepsilon)\right)$ we denote a matrix consisting of elements infinitely small with respect to $\varepsilon$. Because the elements of the matrix $\bar{D}_{0}$ are continuous for all $\varepsilon \in\left(0, \varepsilon_{0}\right]$ and $\lim _{\varepsilon \rightarrow 0} D_{0}(\varepsilon)=\bar{D}_{0}$, then we determine the matrix $D_{0}(\varepsilon)$ for $\varepsilon=0$, putting $D_{0}(0)=\bar{D}_{0}$. We neglect the exponentially small elements in the matrix $D_{0}(\varepsilon)$ and system (2.14) takes the form

$$
M\left(\begin{array}{c}
c_{0} \\
\eta_{0}
\end{array}\right)=h_{0}
$$

where $M=\left[\bar{D}_{0}, S_{0}\right]$ is a $(m \times(n+p-k-s))$ constant matrix.

Let the following condition hold:

(H5) $\operatorname{rank} M=m=n-k+p-s$.

Then $\operatorname{det} M \neq 0$ and system (2.15) is always solvable and

$$
\begin{aligned}
& c_{0}=\left[M^{-1}\right]_{n-k} h_{0} \\
& \eta_{0}=\left[M^{-1}\right]_{p-s} h_{0},
\end{aligned}
$$

where $\left[M^{-1}\right]_{n-k}$ and $\left[M^{-1}\right]_{p-s}$ are the first $(n-k)$ and last $(p-s)$ rows of the matrix $M^{-1}$. We should note that in this case $n-m=k-p+s>0$, i. e., $n>m$. 
We substitute (2.16) into (2.13) and (2.9) and get

$$
\begin{aligned}
x_{0}(t) & =P_{A_{p}} \Phi(t, a)\left[M^{-1}\right]_{p-s} h_{0}+\bar{x}_{0}(t), \\
\Pi_{0}(\tau) & =X_{n-k}(\tau)\left[M^{-1}\right]_{n-k} h_{0},
\end{aligned}
$$

where $\bar{x}_{0}(t)=P_{A_{p}} \bar{\alpha}_{0}(t)-A^{\dagger} \varphi(t)$.

2.2. Obtaining the coefficients $x_{1}(t)$ and $\Pi_{1}(\tau)$. To obtain the coefficient $x_{1}(t)$ from (2.10), it is sufficient to determine the function $\alpha_{1}(t)$. This will be realized in terms of the coefficient $x_{2}(t)$. System (2.3) under $i=2$ has a solution $x_{2}(t)=$ $P_{A_{p}} \alpha_{2}(t)+A^{\dagger} L_{1}\left(x_{1}(t)\right)$ if and only if

$$
P_{A_{p}^{*}} L_{1}\left(x_{1}(t)\right)=0
$$

for all $t \in[a, b]$. In the last equation we substitute $x_{1}(t)$ from (2.10). Keeping in mind the form of the operator $L_{1}$, for determining the function $\alpha_{1}(t)$, we obtain the degenerate differential system

$$
C \frac{d}{d t} \alpha_{1}(t)=B(t) \alpha_{1}(t)+g_{1}(t), \quad t \in[a, b],
$$

where $g_{1}(t)=-P_{A_{p}^{*}} L_{1}\left(A^{\dagger} L_{1} x_{0}(t)\right)$.

System (2.18) coincides with system (2.3), (1.4) at $l(t) \equiv g_{1}(t), t \in[a, b]$ and in accordance with Lemma 1.2 and equation (1.9), we obtain

$$
\alpha_{1}(t)=\Phi(t, a) \eta_{1}+\bar{\alpha}_{1}(t), \quad t \in[a, b], \quad \eta_{1} \in \mathbb{R}^{p-s},
$$

where $\bar{\alpha}_{1}(t)=Q_{1}(t) \bar{u}_{1}(t)-Q_{2}(t) v_{1}(t)$,

$$
\bar{u}_{1}(t)=\Phi(t) \int_{a}^{t} \Phi^{-1}(s) \mu_{1}(s)_{0} d s,
$$

and $P(t) g_{1}(t)=\left(\begin{array}{c}\mu_{1}(t) \\ v_{1}(t)\end{array}\right)$. The vector-functions $u_{1}(t)$ and $v_{1}(t)$ are solutions of system (1.7), where $u(t)=u_{1}(t), v(t)=v_{1}(t)$.

We substitute (2.19) into $x_{1}(t)$ from (2.10) and obtain

$$
x_{1}(t)=P_{A_{p}} \Phi(t, a) \eta_{1}+P_{A_{p}} \bar{\alpha}_{1}(t)+A^{\dagger} L_{1}\left(x_{0}(t)\right) .
$$

In accordance with Lemma 3 , the general solution of the system (2.4) at $i=1$ is

$$
\Pi_{1}(\tau)=X_{n-k}(\tau) c_{1}+\int_{0}^{+\infty} K(\tau, s) \psi_{1}(s) d s, \quad c_{1} \in \mathbb{R}^{n-k} .
$$

We substitute (2.20) and (2.21) into (2.5) at $i=1$. The constant vectors $\eta_{1}$ and $c_{1}$ are obtained by the system

$$
D_{0}(\varepsilon) c_{1}+S_{0} \eta_{1}=h_{1}(\varepsilon)
$$

where

$$
h_{1}(\varepsilon)=-l\left(\int_{0}^{+\infty} K\left(\frac{\cdot-a}{\varepsilon}, s\right) \psi_{1}(s) d s\right)-l\left(P_{A_{p}} \bar{\alpha}_{1}(\cdot)\right)-l\left(A^{\dagger} L_{1}\left(x_{0}(\cdot)\right)\right) .
$$


Obviously,

$$
h_{1}(\varepsilon)=h_{10}+O\left(\varepsilon^{s_{1}} \exp (-\alpha / \varepsilon)\right)
$$

i. e., $h_{1}(\varepsilon)$ is with continuous elements for all $\varepsilon \in\left(\varepsilon_{0}\right]$ and $\lim _{\varepsilon \rightarrow 0} h_{1}(\varepsilon)=h_{10}$. Then we determine $h_{1}(\varepsilon)$ for $\varepsilon=0$, putting $h_{1}(0)=h_{10}$. Since $D_{0}(0)=\bar{D}_{0}$, in system (2.22) we neglect the exponentially small elements and obtain

$$
M\left(\begin{array}{c}
c_{1} \\
\eta_{1}
\end{array}\right)=h_{10},
$$

where $M$ is the matrix from Section 2.1.

In accordance with condition (H5), the solution of the system (2.23)

$$
c_{1}=\left[M^{-1}\right]_{n-k} h_{10}, \quad \eta_{1}=\left[M^{-1}\right]_{p-s} h_{10},
$$

we substitute into (2.20) and (2.21). Consequently, the coefficients $x_{1}(t)$ and $\Pi_{1}(\tau)$ have the form

$$
\begin{aligned}
x_{1}(t) & =P_{A_{p}} \Phi(t, a)\left[M^{-1}\right]_{p-s} h_{10}+\bar{x}_{1}(t), \\
\Pi_{1}(\tau) & =X_{n-k}(\tau)\left[M^{-1}\right]_{n-k} h_{10}+\bar{\Pi}_{1}(\tau),
\end{aligned}
$$

where $\bar{x}_{1}(t)=P_{A_{p}} \bar{\alpha}_{1}(t)+A^{\dagger} L_{1}\left(x_{0}(t)\right)$ and $\bar{\Pi}_{1}(\tau)=\int_{0}^{+\infty} K(\tau, s) \psi_{1}(s) d s$.

2.3. Determining the coefficients $x_{q}(t)$ and $\Pi_{q}(\tau), q>1$. The inductive approach shows that the coefficients $x_{q}(t)$ and $\Pi_{q}(\tau)(q>1)$ have the form

$$
\begin{aligned}
x_{q}(t) & =P_{A_{p}} \Phi(t, a)\left[M^{-1}\right]_{p-s} h_{q 0}+\bar{x}_{q}(t), \\
\Pi_{q}(\tau) & =X_{n-k}(\tau)\left[M^{-1}\right]_{n-k} h_{q 0}+\bar{\Pi}_{q}(\tau),
\end{aligned}
$$

where

$$
\begin{aligned}
h_{q 0} & =\lim _{\varepsilon \rightarrow 0} h_{q}(\varepsilon), \\
h_{q}(\varepsilon) & =-l\left(\int_{0}^{+\infty} K\left(\frac{\cdot-a}{\varepsilon}, s\right) \psi_{q}(s) d s\right)-l\left(P_{A_{p}} \bar{\alpha}_{q}(\cdot)\right)-l\left(A^{\dagger} L_{1}\left(x_{q-1}(\cdot)\right)\right), \\
\bar{x}_{q}(t) & =P_{A_{p}} \bar{\alpha}_{q}(t)+A^{\dagger} L_{1}\left(x_{q-1}(t)\right), \\
\bar{\Pi}_{q}(\tau) & =\int_{0}^{+\infty} K(\tau, s) \psi_{q}(s) d s .
\end{aligned}
$$

Assume that the coefficients $x_{i}(t)$ and $\Pi_{i}(\tau) i=\overline{1, q-1}$ are determined. System (2.3) for $i=q$ has a solution

$$
x_{q}(t)=P_{A_{p}} \alpha_{q}(t)+A^{\dagger} L_{1}\left(x_{q-1}(t)\right)
$$

if and only if $P_{A_{p}^{*}} L_{1}\left(x_{q-1}(t)\right)=0$ for all $t \in[a, b]$. However, this equality is fulfilled because it is used in obtaining the function $\alpha_{q-1}(t)$. This solution $\alpha_{q-1}(t)$ participates in $x_{q-1}(t)$, which with respect to the induction hypothesis is determined completely. 
The function $\alpha_{q}(t)$ is obtained from the solvability condition $P_{A_{p}^{*}} L_{1}\left(x_{q}(t)\right)=0$, $\forall t \in[a, b]$ of system (2.3) for $i=q+1 A x_{q+1}(t)=L_{1}\left(x_{q}(t)\right)$. Thus, we obtain the following differential system (see (1.4)):

$$
C \frac{d}{d t} \alpha_{q}(t)=B(t) \alpha_{q}(t)+g_{q}(t), \quad t \in[a, b],
$$

where $g_{q}(t)=-P_{A_{p}^{*}} L_{1}\left(A^{\dagger} L_{1}\left(x_{q}(t)\right)\right)$.

By Lemma 2 and equation (1.9) we find

$$
\alpha_{q}(t)=\Phi(t, a) \eta_{q}+\bar{\alpha}_{q}(t), \quad t \in[a, b], \quad \eta_{q} \in \mathbb{R}^{p-s},
$$

where $\bar{\alpha}_{q}(t)=Q_{1}(t) \bar{u}_{q}(t)-Q_{2}(t) v_{q}(t)$,

$$
\bar{u}_{q}(t)=\Phi(t) \int_{a}^{t} \Phi^{-1}(s) \mu_{q}(s)_{0} d s
$$

and $P(t) g_{q}(t)=\left(\begin{array}{c}\mu_{q}(t) \\ v_{q}(t)\end{array}\right)$.

The vector-functions $u_{q}(t)$ and $v_{q}(t)$ are solutions of system (1.7), where $u(t)=$ $u_{q}(t), v(t)=v_{q}(t)$.

We substitute (2.28) into (2.27) and obtain

$$
x_{q}(t)=P_{A_{p}} \Phi(t, a) \eta_{q}+P_{A_{p}} \bar{\alpha}_{q}(t)+A^{\dagger} L_{1}\left(x_{q-1}(t)\right), \quad \eta_{q} \in \mathbb{R}^{p-s} .
$$

The general solution of system (2.4) for $i=q$ is

$$
\Pi_{q}(\tau)=X_{n-k}(\tau) c_{q}+\int_{0}^{+\infty} K(\tau, s) \psi_{q}(s) d s, \quad c_{q} \in \mathbb{R}^{n-k} .
$$

We substitute (2.29) and (2.30) in the boundary condition (2.5) for $i=q$ and get the system

$$
D_{0}(\varepsilon) c_{q}+S_{0} \eta_{q}=h_{q}(\varepsilon)
$$

where

$$
h_{q}(\varepsilon)=-l\left(\int_{0}^{+\infty} K\left(\frac{\cdot-a}{\varepsilon}, s\right) \psi_{q}(s) d s\right)-l\left(P_{A_{p}} \bar{\alpha}_{q}(\cdot)\right)-l\left(A^{\dagger} L_{1}\left(x_{q-1}(\cdot)\right)\right) .
$$

Since

$$
D_{0}(\varepsilon)=\bar{D}_{0}+O\left(\varepsilon^{s} \exp (-\alpha / \varepsilon)\right)
$$

$\bar{D}_{0}=\lim _{\varepsilon \rightarrow 0} D_{0}(\varepsilon), h_{q}(\varepsilon)=h_{q 0}+O\left(\varepsilon^{s_{1}} \exp (-\alpha / \varepsilon)\right)$, and $h_{q 0}=\lim _{\varepsilon \rightarrow 0} h_{q}(\varepsilon)$, after ignoring the exponentially small elements, the last system takes the form

$$
M\left(\begin{array}{c}
c_{q} \\
\eta_{q}
\end{array}\right)=h_{q 0}
$$

with the solution (see (H5))

$$
c_{q}=\left[M^{-1}\right]_{n-k} h_{q 0}, \quad \eta_{q}=\left[M^{-1}\right]_{p-s} h_{q 0} .
$$

We substitute (2.31) in (2.29) and (2.30) and obtain the equations (2.25), (2.26). 
All the boundary functions $\Pi_{i}(\tau)$ satisfy inequalities (2.2). This follows from Lemma 3 and the inequality

$$
\left\|X_{n-k}(\tau)\right\| \leq c_{1} \exp \left(-\beta_{1} \tau\right)
$$

where $c_{1}>0, \beta_{1}>0$, and $\tau>0$. After sequential analysis we get

$$
\left\|\Pi_{0}(\tau)\right\| \leq\left\|X_{n-k}(\tau)\right\|\left\|\left[M^{-1}\right]_{n-k}\right\|\left\|h_{0}\right\| \leq c_{1} \exp \left(-\beta_{1} \tau\right) c_{2} c_{3}=\gamma_{0} \exp \left(-\alpha_{0} \tau\right),
$$

where $\left\|\left[M^{-1}\right]_{n-k}\right\| \leq c_{2},\left\|h_{0}\right\| \leq c_{30}, \gamma_{0}=c_{1} c_{2} c_{30}, \alpha_{0}=\beta_{1}$, and

$$
\begin{aligned}
& \left\|\Pi_{1}(\tau)\right\| \leq\left\|X_{n-k}(\tau)\right\|\left\|\left[M^{-1}\right]_{n-k}\right\|\left\|h_{10}\right\|+\left\|\bar{\Pi}_{1}(\tau)\right\| \leq \\
& \leq c_{1} \exp \left(-\beta_{1} \tau\right) c_{2} c_{31}+\bar{c}_{1} \exp \left(-\bar{\beta}_{1} \tau\right) \\
& \leq\left(c_{1} c_{2} c_{31}+\bar{c}_{1}\right) \exp \left(-\alpha_{1} \tau\right)=\gamma_{1} \exp \left(-\alpha_{1} \tau\right),
\end{aligned}
$$

where $\mid h_{10}\left\|\leq c_{31},\right\| \bar{\Pi}_{1}(\tau) \| \leq \bar{c}_{1} \exp \left(-\bar{\beta}_{q} \tau\right)$, and $\alpha_{1}=\max \left(\beta_{1}, \bar{\beta}_{1}\right)$. Finally,

$$
\begin{aligned}
& \left\|\Pi_{q}(\tau)\right\| \leq\left\|X_{n-k}(\tau)\right\|\left\|\left[M^{-1}\right]_{n-k}\right\|\left\|\mid h_{q 0}\right\|+\left\|\bar{\Pi}_{q}(\tau)\right\| \leq \\
& \leq c_{1} \exp \left(-\beta_{1} \tau\right) c_{2} c_{3 q}+\bar{c}_{q} \exp \left(-\bar{\beta}_{q} \tau\right) \\
& \quad \leq\left(c_{1} c_{2} c_{3 q}+\bar{c}_{q}\right) \exp \left(-\alpha_{q} \tau\right)=\gamma_{q} \exp \left(-\alpha_{q} \tau\right),
\end{aligned}
$$

where $\mid h_{q 0}\left\|\leq c_{3 q},\right\| \bar{\Pi}_{q}(\tau) \| \leq \bar{c}_{q} \exp \left(-\bar{\beta}_{q} \tau\right)$, and $\alpha_{q}=\max \left(\beta_{1}, \bar{\beta}_{q}\right)$. Thus, the following theorem is true.

Theorem 1. Let conditions $(\mathrm{H} 1)-(\mathrm{H} 5)$ hold and let $\operatorname{det} B_{11}(t) \neq 0$. Then the boundary-value problems (1.1), (1.2) have a formally asymptotic solution of form (2.1). The coefficients of the regular and singular series have representations (2.17) and (2.25) for $q=1,2, \ldots$ For the boundary functions, the following estimate holds:

$$
\left\|\Pi_{q}(\tau)\right\| \leq \gamma_{q} \exp \left(-\alpha_{q} \tau\right), \quad q=0,1,2, \ldots,
$$

where $\gamma_{q}$ and $\alpha_{q}$ are positive constants. Moreover, the equality

$$
\lim _{\varepsilon \rightarrow 0} x(t, \varepsilon)=x_{0}(t)
$$

holds for $t \in(a, b]$.

Remark 1. The case where $\operatorname{rank} M=n_{1}<\min (m, n-k+p-s)$ and $p=s$ is of independent interest.

\section{A BOUND OF THE REMAINDER TERM OF THE ASYMPTOTIC SERIES}

The solution of the boundary-value problem (1.1), (1.2) we seek in the form

$$
x(t, \varepsilon)=X_{n}(t, \varepsilon)+u_{n}(t, \varepsilon),
$$

where $X_{n}(t, \varepsilon)=\sum_{i=0}^{n} \varepsilon^{i}\left(x_{i}(t)+\Pi_{i}(\tau)\right), \tau=\frac{t-a}{\varepsilon}, t \in[a, b]$.

We shall prove that, for $t \in[a, b]$ and $\varepsilon \in\left(0, \varepsilon_{0}\right]$, the function $u_{n}(t, \varepsilon)$ satisfies the inequality $\left\|u_{n}(t, \varepsilon)\right\| \leq K \varepsilon^{n+1}$, where $K>0$ and $\lim _{\varepsilon \rightarrow 0} x(t, \varepsilon)=x_{0}(t)$. 
Let the smoothness degree of the elements of the matrix $A_{1}(t)$ and the function $\varphi(t)$ is $n+2$.

If $u_{n}(t, \varepsilon)=\varepsilon^{n+1}\left(x_{n+1}(t)+\Pi_{n+1}\right)+u_{n+1}(t, \varepsilon)$ and we should prove that $\left\|u_{n+1}(t, \varepsilon)\right\| \leq$ $\bar{K} \varepsilon^{n+1}, \bar{K}>0$, then there would exist a positive constant $K$ such that $\left\|u_{n}(t, \varepsilon)\right\| \leq$ $K \varepsilon^{n+1}$.

Substituting $x(t, \varepsilon)=X_{n+1}(t, \varepsilon)+u_{n+1}(t, \varepsilon)$ in problem (1.1), (1.2), for the determination of $u_{n+1}(t, \varepsilon)$, we get the boundary-value problem

$$
\begin{gathered}
\varepsilon \frac{d u_{n+1}(t, \varepsilon)}{d t}=A u_{n+1}(t, \varepsilon)+G\left(t, u_{n+1}, \varepsilon\right), \\
l\left(u_{n+1}(\cdot, \varepsilon)\right)=0 .
\end{gathered}
$$

The function $G\left(t, u_{n+1}, \varepsilon\right)$ has the form

$$
\begin{aligned}
& G\left(t, u_{n+1}(t, \varepsilon), \varepsilon\right)=A X_{n+1}(t, \varepsilon)+\varepsilon A_{1}(t, \varepsilon)\left[X_{n+1}(t, \varepsilon)+u_{n+1}(t, \varepsilon)\right]+ \\
&+\varphi(t)-\varepsilon \frac{d X_{n+1}(t, \varepsilon)}{d t}
\end{aligned}
$$

and satisfies the following conditions:

I. $\|G(t, 0, \varepsilon)\| \leq \xi \varepsilon^{m+2}$, where $\xi>0$;

II. For all $\eta>0$, a exists $\delta=\delta(\eta)$ and $\varepsilon_{0}=\varepsilon_{0}(\eta)$ such that if $\left\|u_{n+1}^{\prime}\right\| \leq \delta$ and $\left\|u_{n+1}^{\prime \prime}\right\| \leq \delta$, then

$$
\left\|G\left(t, u_{n+1}^{\prime}, \varepsilon\right)-G\left(t, u_{n+1}^{\prime \prime}, \varepsilon\right)\right\| \leq \eta\left\|u_{n+1}^{\prime}-u_{n+1}^{\prime \prime}\right\|
$$

for $t \in[a, b]$ and $0<\varepsilon \leq \varepsilon_{0}$.

Let $A=\operatorname{diag}(\bar{A}, \overline{\bar{A}}), \overline{\bar{A}}=\operatorname{diag}\left(J, \Theta_{p-s}\right)$ is a $(k \times k)$ matrix, $J=\operatorname{diag}\left(J_{1}, \ldots, J_{s}\right)$ is a $((k-p+s) \times(k-p+s))$ matrix. Then we represent $u_{n+1}$ in the form

$$
u_{n+1}(t, \varepsilon)=\left(\omega_{1}(t, \varepsilon), \omega_{2}(t, \varepsilon), \omega_{3}(t, \varepsilon)\right)^{T},
$$

where $\omega_{1}(t, \varepsilon)$ is a $(n-k)$-dimensional vector, $\omega_{2}(t, \varepsilon)$ is a $(k-p+s)$-dimensional vector, and $\omega_{3}(t, \varepsilon)$ is a $(p-s)$-dimensional vector.

We introduce the following notation:

$$
A_{1}(t, \varepsilon)=\left(\begin{array}{ll}
A_{111}(t, \varepsilon) & A_{112}(t, \varepsilon) \\
A_{121}(t, \varepsilon) & A_{122}(t, \varepsilon)
\end{array}\right)
$$

where $A_{111}(t, \varepsilon)$ is a $((n-k) \times(n-k))$ matrix, $A_{112}(t, \varepsilon)$ is a $((n-k) \times k)$ matrix, $A_{121}(t, \varepsilon)$ is a $(k \times(n-k))$ matrix, $A_{122}(t, \varepsilon)$ is a $(k \times k)$ matrix;

$$
A_{112}(t)=\left(\begin{array}{ll}
B_{1}(t) & B_{2}(t)
\end{array}\right), \quad A_{121}(t)=\left(\begin{array}{l}
C_{1}(t) \\
C_{2}(t)
\end{array}\right), \quad A_{122}(t)=\left(\begin{array}{ll}
D_{11}(t) & D_{12}(t) \\
D_{21}(t) & D_{22}(t)
\end{array}\right),
$$

where $B_{1}(t)$ is a $((n-k) \times(k-p+s))$ matrix, $B_{2}(t)$ is a $((n-k) \times(p-s))$ matrix, $C_{1}(t)$ is a $((k-p+s) \times(n-k))$ matrix, $C_{2}(t)$ is a $((p-s) \times(n-k))$ matrix, $D_{11}(t)$ is 
a $((k-p+s) \times(k-p+s))$ matrix, $D_{12}(t)$ is a $((k-p+s) \times(p-s))$ matrix, $D_{21}(t)$ is a $((p-s) \times(k-p+s))$ matrix, $D_{22}(t)$ is a $((p-s) \times(p-s))$ matrix;

$$
G(t, 0,0, \varepsilon)=\left(\begin{array}{l}
G_{1}(t, 0,0,0, \varepsilon) \\
G_{2}(t, 0,0,0, \varepsilon) \\
G_{3}(t, 0,0,0, \varepsilon)
\end{array}\right)
$$

where $G_{1}(t, 0,0,0, \varepsilon)$ is a $(n-k)$-dimensional vector, $G_{2}(t, 0,0,0, \varepsilon)$ is a $(k-p+s)$ dimensional vector, $G_{3}(t, 0,0,0, \varepsilon)$ is a $(p-s)$-dimensional vector.

System (3.2) takes the form

$$
\begin{aligned}
\varepsilon \frac{d \omega_{1}}{d t} & =\bar{A} \omega_{1}+\varepsilon A_{111}(t) \omega_{1}+\varepsilon B_{1}(t) \omega_{2}+\varepsilon B_{2}(t) \omega_{3}+G_{1}(t, 0,0,0, \varepsilon), \\
\varepsilon \frac{d \omega_{2}}{d t} & =\left(J+\varepsilon D_{11}(t)\right) \omega_{2}+\varepsilon D_{12}(t) \omega_{3}+\varepsilon C_{1}(t) \omega_{1}+G_{2}(t, 0,0,0, \varepsilon), \\
\varepsilon \frac{d \omega_{3}}{d t} & =\varepsilon D_{21}(t) \omega_{2}+\varepsilon D_{22}(t) \omega_{3}+\varepsilon C_{2}(t) \omega_{1}+G_{3}(t, 0,0,0, \varepsilon) .
\end{aligned}
$$

Obviously, the inequalities $\left\|G_{i}(t, 0,0,0, \varepsilon)\right\| \leq c_{1 i} \varepsilon^{n+2}, c_{1 i}>0, i=1,2,3$, hold on $[a, b]$.

Let $W(t, s, \varepsilon)$ and $V(t, s)$ be the fundamental matrices for the homogeneous systems $\varepsilon \dot{x}=\bar{A} x$ and $\dot{x}=D_{22} x$. Here, $W(s, s, \varepsilon)=E_{n-k}$ and $V(s, s)=E_{p-s}$ are the unit matrices.

Let the Cauchy problem for the homogeneous system $\varepsilon \dot{x}=\left(J+\varepsilon D_{11}(t)\right) x$ have only a trivial solution, and system (3.4) has the particular solution

$$
\omega_{2}(t, \varepsilon)=\int_{a}^{b} K(t, s, \varepsilon)\left[\varepsilon D_{12}(s) \omega_{3}+\varepsilon C_{1}(s) \omega_{1}+G_{2}(s, 0,0,0, \varepsilon)\right] d s, \quad t \in[a, b],
$$

where

$$
K(t, s, \varepsilon)= \begin{cases}\frac{1}{\varepsilon} \bar{X}(t, \varepsilon) \bar{X}^{-1}(s, \varepsilon), & \tau_{i-1} \leq s \leq t \\ 0, & \tau_{i-1} \leq t \leq s,\end{cases}
$$

if the eigenvalues of the matrix $J+\varepsilon D_{11}(t)$ are purely imaginary and

$$
K(t, s, \varepsilon)= \begin{cases}\frac{1}{\varepsilon} \bar{X}(t, \varepsilon) P \bar{X}^{-1}(s, \varepsilon), & \tau_{i-1} \leq s \leq t, \\ -\frac{1}{\varepsilon} \bar{X}(t, \varepsilon)(I-P) \bar{X}^{-1}(s, \varepsilon), & \tau_{i-1} \leq t \leq s,\end{cases}
$$

if the eigenvalues are with a positive or negative real part. The matrix $P$ is a spectral projector of the matrix $J+\varepsilon D_{11}(t)$ on the left half-plane, and $\bar{X}(t, \varepsilon)$ is a normal fundamental matrix for the system $\varepsilon \dot{x}=\left(J+\varepsilon D_{11}(t) x\right.$.

Obviously, $\int_{a}^{b}\|K(t, s, \varepsilon)\| d s \leq \xi_{1}, \xi_{1}>0$, for $t \in[a, b], \varepsilon \in\left(0, \varepsilon_{0}\right]$. 
Lemma 4 ([6,10]). For the matrix $W(t, s, \varepsilon)$, when $a<s \leq t \leq b, 0<\varepsilon \leq \varepsilon_{0}$, the exponential estimate

$$
\|W(t, s, \varepsilon)\| \leq \beta \exp \left(-\alpha\left(\frac{t-s}{\varepsilon}\right)\right), \quad a \leq s \leq t \leq b,
$$

is fulfilled, where $\alpha>0, \beta>0$.

It is clear that $\|V(t, s, \varepsilon)\| \leq \beta_{1}$, where $a \leq s \leq t \leq b, \beta_{1}>0$.

Lemma 5. Any continuous solution of system (3.4)-(3.6) is a solution of the system of integral equations

$$
\begin{aligned}
\omega_{1}(t, \varepsilon)= & W(t, a, \varepsilon) \omega_{1}(a, \varepsilon)+\int_{a}^{t} \frac{1}{\varepsilon}\left[\varepsilon A_{111}(s) \omega_{1}(s, \varepsilon)+\right. \\
& \left.+\varepsilon B_{1}(s) \omega_{2}(s, \varepsilon)+\varepsilon B_{2}(s) \omega_{3}(s, \varepsilon)+G_{1}(s, 0,0,0, \varepsilon)\right] d s, \\
\omega_{2}(t, \varepsilon)= & \int_{a}^{b} K(t, s, \varepsilon)\left[\varepsilon D_{12}(s) \omega_{3}(s, \varepsilon)+\varepsilon C_{1}(s) \omega_{1}(s, \varepsilon)\right. \\
& \left.+G_{2}(s, 0,0,0, \varepsilon)\right] d s, \\
\omega_{3}(t, \varepsilon)= & V(t, a) \omega_{3}(a, \varepsilon)+\int_{a}^{t} V(t, s) \frac{1}{\varepsilon}\left[\varepsilon D_{21}(t) \omega_{2}(s, \varepsilon)+\right. \\
& \left.+\varepsilon D_{22}(s) \omega_{3}+\varepsilon C_{2}(s) \omega_{1}(s, \varepsilon)+G_{3}(s, 0,0,0, \varepsilon)\right] d s .
\end{aligned}
$$

We substitute $u_{n+1}(t, \varepsilon)=\left(\omega_{1}(t, \varepsilon), \omega_{2}(t, \varepsilon), \omega_{3}(t, \varepsilon)\right)^{T}$ into the boundary condition (3.3) and obtain

$$
\bar{l}_{1} \omega_{1}((\cdot), \varepsilon)+\bar{l}_{2} \omega_{2}((\cdot), \varepsilon)+\bar{l}_{3} \omega_{3}((\cdot), \varepsilon)=0,
$$

where $\bar{l}_{i}, i=1,2,3$ are linear $m$-dimensional bounded functionals. After transformations using (3.7)-(3.9), we obtain

$$
\omega_{1}(t, \varepsilon)=W_{i}(t, a, \varepsilon) \omega_{1}(a, \varepsilon)+V_{i}(t, a, \varepsilon) \omega_{3}(a, \varepsilon)+S_{i}\left(t, \omega_{1}, \omega_{3}, a, \varepsilon\right),
$$

$i=1,2,3$, where $W_{1}(t, a, \varepsilon)=W(t, a, \varepsilon)$, and $W_{i}, i=1,2, V_{i}, S_{i}, i=1,2,3$, are functions such that, for all $t \in[a, b]$ and $\varepsilon \in\left(0, \varepsilon_{0}\right]$,

$$
\begin{aligned}
\left\|W_{i}(t, a, \varepsilon)\right\| & \leq \varepsilon k_{i}, \quad k_{i}>0, i=1,2, \\
\| V_{i}(t, a, \varepsilon) & \leq \varepsilon d_{i}, \quad d_{i}>0, i=1,2, \\
\left\|V_{3}(t, a, \varepsilon)\right\| & \leq \beta_{2}+\varepsilon d_{3}, \quad \beta_{2}>0, d_{3}>0, \\
\left\|S_{i}(t, 0,0,0, a, \varepsilon)\right\| & \leq c_{i} \varepsilon^{n+1}, \quad c_{i}>0, i=1,2,3,
\end{aligned}
$$

and

$$
\begin{aligned}
\| S_{i}\left(t, \omega_{1}^{2}, \omega_{3}^{2}, a, \varepsilon\right) & -S_{i}\left(t, \omega_{1}^{1}, \omega_{3}^{1}, a, \varepsilon\right) \| \leq \\
& \leq \varepsilon r_{i} \max _{t \in[a, b]}\left(\left\|\omega_{1}^{2}(t, \varepsilon)-\omega_{1}^{1}(t, \varepsilon)\right\|+\left\|\omega_{3}^{2}(t, \varepsilon)-\omega_{3}^{1}(t, \varepsilon)\right\|\right),
\end{aligned}
$$


where $r_{i}>0, i=1,2,3$. It follows from relation (3.10) that the vector $\omega(a, \varepsilon)=$ $\left(\omega_{1}(a, \varepsilon), \omega_{3}(a, \varepsilon)\right)^{T}$ is determined by the equation

$$
R(\varepsilon) \omega(a, \varepsilon)=q\left(\varepsilon, \omega_{1}, \omega_{3}\right),
$$

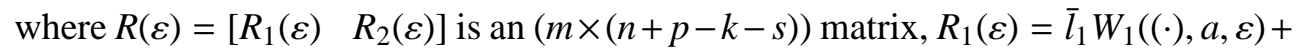
$\bar{l}_{2} W_{2}((\cdot), a, \varepsilon)+\bar{l}_{3} W_{3}((\cdot), a, \varepsilon)$ is an $(m \times(n-k))$ matrix, $R_{2}(\varepsilon)=\bar{l}_{1} V_{1}((\cdot), a, \varepsilon)+$ $\bar{l}_{2} V_{2}((\cdot), a, \varepsilon)+\bar{l}_{3} V_{3}((\cdot), a, \varepsilon)$ is an $(m \times(p-s))$ matrix, and

$$
q\left(\varepsilon, \omega_{1}, \omega_{3}\right)=-\bar{l}_{1} S_{1}\left(\cdot, \omega_{1}, \omega_{3}, a, \varepsilon\right)-\bar{l}_{2} S_{2}\left(\cdot, \omega_{1}, \omega_{3}, a, \varepsilon\right)-\bar{l}_{3} S_{3}\left(\cdot, \omega_{1}, \omega_{3}, a, \varepsilon\right)
$$

is an $m$-dimensional vector. Also, one has $\|q(\varepsilon, 0,0)\| \leq c_{4} \varepsilon^{n+1}, c_{4}>0$, and

$$
\left\|q\left(\varepsilon, \omega_{1}^{2}, \omega_{3}^{2}\right)-q\left(\varepsilon, \omega_{1}^{1}, \omega_{3}^{1}\right)\right\| \leq \varepsilon r_{4} \max _{t \in[a, b]}\left(\left\|\omega_{1}^{2}-\omega_{1}^{1}\right\|+\left\|\omega_{3}^{2}-\omega_{3}^{1}\right\|\right),
$$

where $r_{4}>0$. Since

$$
R(\varepsilon)=R_{0}+O\left(\exp \left(-\frac{\alpha}{\varepsilon}\right)\right)
$$

where $R_{0}$ is a constant matrix, then the following condition is fulfilled:

(H6) $m=n+p-k-s ; \operatorname{det} R(\varepsilon) \neq 0 \forall \varepsilon \in\left[0, \varepsilon_{0}\right]$.

System (3.13) is always solvable and

$$
\begin{aligned}
& \omega_{1}(a, \varepsilon)=\left[R^{-1}\right]_{n-k} q\left(\varepsilon, \omega_{1}, \omega_{3}\right), \\
& \omega_{3}(a, \varepsilon)=\left[R^{-1}\right]_{p-s} q\left(\varepsilon, \omega_{1}, \omega_{3}\right) .
\end{aligned}
$$

We shall substitute (3.14) into (3.7)-(3.9) and obtain a system which will be solved by the method of successive approximations. Let

$$
\begin{aligned}
\omega_{i}^{0}(t, \varepsilon) & =0, \\
\omega_{i}^{s+1}(t, \varepsilon) & =W_{i}(t, a, \varepsilon)\left[R^{-1}\right]_{n-k} q\left(\varepsilon, \omega_{1}^{s}, \omega_{3}^{s}\right)+ \\
& +V_{i}(t, a, \varepsilon)\left[R^{-1}\right]_{p-s} q\left(\varepsilon, \omega_{1}^{s}, \omega_{3}^{s}\right)+S_{i}\left(t, \omega_{1}^{s}, \omega_{3}^{s}, a, \varepsilon\right), \quad i=1,2,3,
\end{aligned}
$$

be the Picard successive approximations.

Theorem 2. Let the conditions of Theorem 1 and assumption (H6) be fulfilled. If $\left\|R^{-1}\right\| \leq c_{R}$, then there exists a positive constant $K$ such that the asymptotic solution of the boundary-value problem (1.1), (1.2) has representation (3.1), where $u_{n}(t, \varepsilon)$ satisfies the inequality

$$
\left\|u_{n}(t, \varepsilon)\right\| \leq K \varepsilon^{n+1} .
$$

Moreover, $x(t, \varepsilon)$ approaches the generating system when $\varepsilon \rightarrow 0$ and $t \in(a, b]$.

Proof. By virtue of (3.10), (3.11), and (3.12), for the first approximation, we have

$$
\max _{t \in[a, b]}\left\|\omega_{i}^{1}(t, \varepsilon)-\omega_{i}^{0}(t, \varepsilon)\right\| \leq K_{i 1}, \quad K_{i 1}>0,
$$

where the constant $K_{i 1} \varepsilon^{n+1}$ is determined by the constants $c_{R}, k_{i}, d_{i}, c_{i}$, and $r_{i}$. 
Let $K^{1}=\max _{i}\left(K_{i 1}\right)$ and $K^{1} \varepsilon^{n+1}=\delta$. For the last approximation we have

$$
\max _{t \in[a, b]}\left\|\omega_{i}^{2}(t, \varepsilon)-\omega_{i}^{1}(t, \varepsilon)\right\| \leq \varepsilon K_{i 2} \delta, \quad K_{i 2}>0, i=1,2,3 .
$$

Let $\varepsilon_{0}=\frac{1}{2} \min _{i}\left(1 / K_{i 2}\right)$. Then

$$
\max _{t \in[a, b]}\left\|\omega_{i}^{2}(t, \varepsilon)-{ }_{i}^{1}(t, \varepsilon)\right\| \leq \frac{1}{2} \delta=\frac{1}{2^{2}} 2 \delta .
$$

Inductively we obtain

$$
\max _{t \in[a, b]}\left\|\omega_{i}^{k+1}(t, \varepsilon)-\omega_{i}^{k}(t, \varepsilon)\right\| \leq \frac{1}{2^{k+1}} 2 \delta .
$$

This reveals that in the segment $[a, b]$, when $\varepsilon$ is sufficiently small, the successive approximations (3.15) are absolutely and uniformly convergent. In addition, we have

$$
\begin{aligned}
\left\|\omega_{i}^{k+1}(t, \varepsilon)\right\| \leq \sum_{j=1}^{k+1}\left\|\omega_{i}^{j}(t, \varepsilon)-\omega_{i}^{j-1}(t, \varepsilon)\right\| & \leq\left(1+\frac{1}{2}+\cdots+\frac{1}{2^{k}}\right) \delta \leq \\
& \leq\left(1+\frac{1}{2}+\cdots+\frac{1}{2^{k}}+\frac{1}{2^{k+1}}+\ldots\right) \delta=2 \delta
\end{aligned}
$$

Let

$$
\lim _{k \rightarrow \infty} \omega_{i}^{k}(t, \varepsilon)=\omega_{i}(t, \varepsilon)
$$

satisfy (3.10) identically. Then, on the interval $[a, b]$, for $\varepsilon \rightarrow 0$, the inequality

$$
\left\|\omega_{i}(t, \varepsilon)\right\| \leq 2 \delta
$$

is fulfilled. Consequently, system (3.10) has an unique continuous solution, which does not escape from the domain $\{(t, \omega) \mid a \leq t \leq b,\|\omega\| \leq 2 \delta\}$. Then, for all $t \in[a, b]$ and $\varepsilon \in\left(0, \varepsilon_{0}\right]$,

$$
\left\|u_{n+1}(t, \varepsilon)\right\| \leq \sum_{i=1}^{3}\left\|\omega_{i}(t, \varepsilon)\right\| \leq 6 \delta=6 K^{1} \varepsilon^{n+1},
$$

i. e., there exists a positive constant $K$ such that the inequality

$$
\left\|u_{n}(t, \varepsilon)\right\| \leq K \varepsilon^{n+1}
$$

is fulfilled and

$$
\lim _{\varepsilon \rightarrow 0} x(t, \varepsilon)=x_{0}(t)
$$

for all $t \in[a, b]$. 


\section{REFERENCES}

[1] Boichuk, A. A. And SAmoilenko, A. M.: Generalized Inverse Operators and Fredholm Boundary Value Problems, VSP, Utrecht-Boston, 2004.

[2] Campbell, S. L.: A general form for solvable linear time varying singular systems of differential equations, SIAM J. Math. Anal. 4 (1987), No. 1101-1115.

[3] Chistyakov, V. F.: On the methods for solving singularity linear systems ordinary differential equations, In: Singularly Perturbed Systems of Ordinary Differential Equations [in Russian], Nauka, Novosibirsk, 1982, pp. 37-65.

[4] Nashed, M. Z.: Generalized Inverses and Applications, Academic Press, New York, San Francisco, London, 1967.

[5] Karandzhulov, L. I.: Asymptotic solution of definite class of singularly perturbed linear boundary-value problems for ordinary differential equations, Annuaire de l'Université de Sofia "St. Kl. Ohridski", Faculté de mathématiques et informatique, Livre 1 - Mathématiques et Mécanique, 91 (1997), 79-95.

[6] Levinson, N.: A boundary value problem for a singularly perturbed differential equation, Duke Math. J., 25 (1958), 331-342.

[7] Penrose, R.: A generalize inverse for matrices, Proc. Cambridge Phil. Soc., 51 (1955), 406-413.

[8] Samoilenko, A. M., Boichuk, A. A., and Karandzhulov, L. I.: Noether boundary value problem with singular perturbation, Diferents. Uravn., 37 (2001), No. 9, 1186-1193.

[9] Samollenko, A. M. and Yakovetz, V. P.: About transformation of linear systems into central canonical form, Dokl. Acad. Nauk Ukr. (1993), No. 4, 10-15.

[10] Vasil'eva, A. B. and Butuzov, V. F.: Singularly Perturbed Equations in the Critical Case [in Russian], Moscow State University, Moscow, 1978.

\section{Author's address}

\section{Lyudmil Karandzhulov:}

Technical University-Sofia, Faculty of Applied Mathematics and Informatics, P. O. Box 384, Sofia 1000, Bulgaria

E-mail address: 1ikar@tu-sofia.bg 